\title{
Volumetric Distribution of the White Matter Hyper-Intensities in Subject with Mild to Severe Carotid Artery Stenosis: Does the Side Play a Role?
}

\author{
Luca Saba, MD, ${ }^{*}$ Pierleone Lucatelli, PhD,† Michele Anzidei, PhD, $\ddagger$ \\ Michele di Martino, PhD, $\neq$ Jasjit S. Suri, PhD, II \#,** and Roberto Montisci, MD††
}

\begin{abstract}
Purpose: The purpose of this paper was to assess the difference in the distribution of white matter hyperintensities (WMHs) on left and right sides of the brain hemispheres of subjects with mild to severe carotid artery stenosis. Material and Methods: Eighty consecutive patients (mean age $71 \pm 6$ years, males 66) with carotid artery stenosis were prospectively recruited. FLAIR-WMH lesion volume was performed using a semiautomated segmentation technique (Jim, Xinapse System, Leicester, UK). The Wilcoxon test was applied to verify the differences in the volume of WMHs between the right and left hemispheres. Results: A statistically significant difference was found in the middle cerebral artery (MCA) territory for the volume of the lesions (median volume of WMHs of the left side $=889.5 \mathrm{~mm}^{3}$; median volume of WMHs on the right side $=580.5 \mathrm{~mm}^{3} ; P=.0416$ ); no statistically significant difference was found on the other territories by taking into considerations the lesions. By analyzing the degree of stenosis, we found a higher degree of stenosis of the left side $(67.9 \%$; $95 \%$ confidence interval [CI], 64.8\%-70.9\%) compared with the right side $(65.7 \%$; $95 \% \mathrm{CI}, 62.4 \%-68.9 \%)$, but the Mann-Whitney test did not show a statistically significant difference $(P=.3235)$. Conclusions: Results of our study suggest that there is a difference in the distribution of WMHs in the brain hemispheres according to the left/right side on the MCA territories and for the periventricular white matter in subjects with mild to severe carotid artery stenosis. Key Words: Carotid artery-white matter-leukoaraiosis-magnetic resonance. (C) 2018 National Stroke Association. Published by Elsevier Inc. All rights reserved.
\end{abstract}

From the ${ }^{*}$ Department of Radiology, University Hospital of Cagliari, Italy, SS554 Monserrato, CA, Italy; +Vascular and Interventional Radiology Unit; ‡Department of Radiology, Sapienza University of Rome, Viale Regina Elena, 324, 00161, Rome, Italy; IMonitoring and Diagnostic Division, AtheroPoint ${ }^{\mathrm{TM}}$, Roseville, CA, USA; \#Point-ofCare Devices, Global Biomedical Technologies, Inc., Roseville, CA, USA; **Department of Electrical Engineering, University of Idaho (Aff.), ID, USA; and t+Department of Vascular Surgery, University Hospital of Cagliari, Italy, SS554 Monserrato, CA, Italy.

Received January 11, 2018; revision received February 16, 2018; accepted February 28, 2018.

Address correspondence to Luca Saba, MD, AOU, Cagliari, Italy.

E-mail: lucasaba@tiscali.it.

$1052-3057 / \$$ - see front matter

(C) 2018 National Stroke Association. Published by Elsevier Inc. All rights reserved.

https://doi.org/10.1016/j.jstrokecerebrovasdis.2018.02.065

\section{Introduction}

The term "white matter hyperintensities" (WMHs) indicates the presence of hyperintensities on T2-weighted images in the white matter. ${ }^{1} \mathrm{WMH}$ is commonly encountered in the brain of elderly patients ${ }^{1}$ and in the general population. Its prevalence ranges from $11 \%$ to $21 \%$ in adults aged around 64 years to $94 \%$ at the age of 82 years., ${ }^{2,3}$

Previously pathology-published studies found that those areas where $\mathrm{WMH}$ is detected are characterized by the presence of tissue rarefaction associated with loss of myelin and axons; in some cases, findings show a moderate degree of gliosis. ${ }^{4,5}$

A higher prevalence of $\mathrm{WMH}$ was found in subjects with hypertension and cerebrovascular risk factors, ${ }^{6-8}$ 
and this parameter is commonly used to assess cerebrovascular burden in cognitive impairment ${ }^{9}$ and appears to be associated with an increased risk of cognitive decline. ${ }^{10}$

In the past years the WMH presence and degree of severity were assessed with qualitative scales ${ }^{11}$ based on the subjective assessment of the observer, whereas nowadays, thanks to the development of automated and semiautomated software, it is possible to quantify the volume of $\mathrm{WMHs}^{12,13}$ in cubic millimeters.

In the last years, studies have demonstrated that a statistically significant higher prevalence of cerebrovascular ischemic events involves the left hemisphere more than the right ${ }^{14-16}$ by showing that the left side plays a significant role in the risk of developing ischemic events. Recently, a study showed that carotid atherosclerotic plaque size and composition (in particular the intraplaque hemorrhage) are not symmetrically distributed, with the leftsided plaques being more vulnerable. ${ }^{17}$

We aimed to assess the volumetric distribution of the WMHs in the left and right hemispheres according to the different arterial territories (anterior cerebral artery [ACA], middle cerebral artery [MCA], and posterior cerebral artery [PCA] $)$ and to the involvement of periventricular white matter (PVWM) or deep white matter (DWM).

\section{Material and Methods}

\section{Study Design and Patient Population}

The Institutional Review Board approval for this study was obtained, and patients' consent was waived because of the retrospective nature of the study. Based on a power calculation (type I error, $\alpha=.05$; type II error $\beta=.1$; Az Null Hypothesis value $=.5 ; \mathrm{Az}$ significant value $=.7$, pooled group), we estimated that a sample size of at least 145 hemispheres would be sufficient to investigate the potential difference in the volumetric distribution of the WHMs in the left and right hemispheres according to the different arterial territories. We decided to also include a correction of $10 \%$, and therefore, we included 80 consecutive patients (for 160 brain hemispheres) who underwent brain magnetic resonance imaging (MRI) in our hospital form March 2012 to December 2013 (66 men, 14 women; mean age, 71 years; age range $48-83$ years).

Patient selection restricted to persons with carotid atherosclerosis and the subjects were selected by our database by considering those subjects aged more than 40 years who had a brain MRI because of the presence of atherosclerotic disease in the carotid arteries documented by using ultrasonography. In our institute those patients who showed an atherosclerotic disease that determines a stenosis degree $>30 \%$ according to the North American Symptomatic Carotid Endarterectomy Trial criteria ${ }^{18}$ were invited to undergo an MRI exam of the brain.

To quantify the stenosis degree using CTA, oblique axial images normal to ICA lumen centerline were elabo- rated and the value was calculated by comparing the diameter of the stenosed segment with the most distal normal one, where no stenosis was present.

We considered the following as exclusion criteria: (1) other possible etiologies for white matter disease, such as vasculitis, demyelinating diseases, and connective tissue diseases; (2) concomitant pathologies such as brain tumor, abscess, and encephalitis; (3) strokes (as reported by clinical charts with brain MRI/computed tomography confirmation of stroke); (4) the presence of cardioembolism documented by cardiologists; and (5) absolute MRI contraindications.

Part of the examined population was included in previously published studies.

\section{Brain MRI Technique}

Brain MRI examinations were performed according to a previously described technique ${ }^{19}$ on a Gyroscan 1.5-T MRI scanner (Philips, Best, The Netherlands) with a head coil. As part of our general brain protocol, axial and sagittal 2D FLAIR images (10,000/140/2200 msec for TR/ TE/TI; matrix: $512 \times 512$; FOV: $240 \times 240 \mathrm{~mm}^{2}$; section thickness: $5 \mathrm{~mm}$ ) were obtained and used for the determination of WMH volume. Also T1-weigheted and T2weigheted images were considered in order to exclude chronic silent brain infarcts.

\section{MRI WMH Volume and Number Analysis}

The volumetric measurement of the WMHs was performed using a semiautomated segmentation technique (Jim, Xinapse System, Leicester, United Kingdom). The axial FLAIR images were selected, and 1 experienced neuroradiologist performed the analysis by considering hyperintense white matter regions on FLAIR images not related to large vessel infarcts as WMHs. The neuroradiologist was blinded for the main purpose of this study and to the clinical data. After the delineation of WMHs, the WMH volumes for each hemisphere were automatically produced by the software, based on the slice thickness and outlined WMH areas. The number of lesions for each hemisphere was calculated. Moreover, the same neuroradiologist classified the WMHs according to the different arterial territories (ACA, MCA, and PCA) and to the involvement of the PVWM or the DWM.

The regions assessed for WMHs were the frontal, parietal, occipital, and temporal lobes, basal ganglia, and infratentorial regions. DWM included frontal, parietal, occipital, and temporal regions, whereas PVWM included lesions in 3 regions (frontal and occipital caps and bands), and a periventricular lesion was operationally defined as adjacent to the ventricle.

Lacunar silent brain infarcts were excluded from the analysis and were identified according to Vermeer et al's $^{20}$ classification, in whom lacunar infarctions were defined as well-defined small focal lesions, most commonly located 
Table 1. Patient Demographics

\begin{tabular}{lc}
\hline \multicolumn{1}{c}{ Age $^{*}$} & $71 \pm 6$ years $^{\dagger}$ \\
\hline Sex (male) & $\mathrm{n}=66(82.5 \%)$ \\
Smoker & $\mathrm{n}=61(76.2 \%)$ \\
Hypertension & $\mathrm{n}=42(52.5 \%)$ \\
CAD & $\mathrm{n}=18(22.5 \%)$ \\
Diabetes & $\mathrm{n}=25(31.2 \%)$ \\
Statins and other drugs & ${ }^{\dagger}$ \\
Dyslipidemia & $\mathrm{n}=45(56.2 \%)$ \\
Cholesterol total & $112 \pm 60$ \\
Cholesterol HDL & $176 \pm 48$ \\
\hline
\end{tabular}

Abbreviations: CAD, coronary artery disease; HDL, high density lipoprotein; SD, standard deviation.

*Mean age.

$\dagger$ Other lipid-lowering drugs.

in the deep brain with roughly the same intensity as cerebrospinal fluid on MRI. Dilated perivascular spaces can mimic lacunar infarcts, and we distinguish them from lacunar infarctions based on the size $(<3 \mathrm{~mm})$, shape (round or linear), and location (lower basal ganglia region). Cerebral micro-bleeds were also excluded from the analysis, and they were considered as focal, rounded, or circular areas of very low signal intensity that were smaller than $10 \mathrm{~mm}$ in size according to a previous paper by Jeerakathil et al. ${ }^{21}$

\section{Statistical Analysis}

The normality of each continuous variable group was tested using the Kolmogorov-Smirnov $Z$ test. Continuous data were described as the mean value \pm standard deviation (SD), whereas non-Gaussian with median and percentiles. The Mann-Whitney test was applied to test the difference between the degree of stenosis in the right/ left carotid artery. The Wilcoxon test was applied to verify the differences in the volume of WMHs between the right and left hemispheres. Pearson rho correlation analysis was performed to evaluate the association between the $\mathrm{WMH}$ volume of the different territories, and scatter plots were generated. A $P$ value $<.05$ was regarded to indicate a statistically significant association. All $P$ values were calculated using a 2-tailed significance level. Statistical analysis was performed with the SPSS 13.0 statistical package (SPSS Inc., Chicago, IL). Graphics were plotted with MedCalc 15.0 software (MedCalc, Mariakerke, Belgium).

\section{Results}

\section{General Results}

No patients were excluded from the analysis, and all the subjects were asymptomatic. The clinical characteristics of the population we studied are summarized in Table 1, whereas in Table 2 the summary of $\mathrm{WMH}$ volume and lesions are given. By analyzing the degree of stenosis, we found a higher degree of stenosis of the left side (67.9\%; 95\% confidence interval, 64.8\%-70.9\%) compared with the right side $(65.7 \% ; 95 \%$ confidence interval, $62.4 \%-68.9 \%$ ), but the Mann-Whitney test did not show any statistically significant difference $(P=.3235)$. The median $W M H$ volume was $3505.5 \mathrm{~mm}^{3}$. The median WMH volume was $1289 \mathrm{~mm}^{3}$ (SD $2279 \mathrm{~mm}^{3}$ ) in the right hemisphere and $1760 \mathrm{~mm}^{3}\left(\mathrm{SD}, 2231 \mathrm{~mm}^{3}\right)$ in the left hemisphere $(P=.0899)$. The median number of lesions in the right hemisphere was 15, whereas the median number in the left hemisphere was $15(P$ value $=.0847)$. According to the Kolmogorov-Smirnov $Z$ test the data were non-Gaussian, and nonparametric tests were accordingly performed.

Table 2. Summary of WMH volume and number of lesions

\begin{tabular}{|c|c|c|c|c|c|}
\hline & Median & Minimum & Maximum & 5-95 percentiles & $\begin{array}{l}\text { Normal distribution } \\
\qquad(P \text { value })^{\ddagger}\end{array}$ \\
\hline WMH total volume & 3505.5 & 0 & 28,324 & $306.000-11,549.500$ & $<.0001$ \\
\hline WMH total infratentorial volume & 32 & 0 & 178 & $5.000-82.000$ & $<.0001$ \\
\hline WMH total supratentorial volume & 3170 & 0 & 27,672 & $306.000-11,549.500$ & $<.0001$ \\
\hline Total brain number of lesion & 32.5 & 0 & 180 & $4.500-82.000$ & $<.0001$ \\
\hline Total supratentorial number of lesions & 32 & 0 & 178 & $5.000-82.000$ & $<.0001$ \\
\hline Total infratentorial number of lesions & 0 & 0 & 8 & $.000-6.000$ & .0012 \\
\hline WMH volume of right hemisphere* & 1289 & 0 & 14,400 & $24.500-5414.000$ & $<.0001$ \\
\hline WMH volume of left hemisphere* & 1760 & 0 & 13,272 & $126.000-6741.000$ & $<.0001$ \\
\hline Number of lesions in the right hemisphere ${ }^{\dagger}$ & 15 & 0 & 55 & $1.000-42.000$ & .0077 \\
\hline Number of lesions in the left hemisphere & 15.5 & 0 & 167 & $2.500-42.500$ & $<.0001$ \\
\hline
\end{tabular}

Abbreviation: WMH, white matter hyperintensity.

*Wilcoxon test; $P=.0899$.

$\dagger$ Wilcoxon test; $P=.0847$.

$\$$ Normal distribution assessed with the Kogomorov-Smirnov $\mathrm{Z}$ test. 
Table 3. Summary of $W M H$ volume and number of lesions in $A C A-M C A-P C A$

\begin{tabular}{lccccc} 
& & & & & Normal distribution \\
& Median & Minimum & Maximum & 5-95 percentiles & $<$ * \\
\hline ACA lesions left & 4 & 0 & 153 & $0-15$ & $<.0001$ \\
ACA lesions right & 4 & 0 & 24 & $0-14.5$ & $<.0001$ \\
ACA lesions total & 8.5 & 0 & 157 & $0-27.5$ & $<.0001$ \\
ACA volume left & 282.5 & 0 & 4180 & $0-1408$ & $<.0001$ \\
ACA volume right & 242.5 & 0 & 5845 & $0-1696.5$ & $<.0001$ \\
ACA volume total & 538 & 0 & 8964 & $0-2678$ & $<.0001$ \\
MCA lesions left & 7 & 0 & 33 & $1-22.5$ & $<.0001$ \\
MCA lesions right & 7 & 0 & 31 & $0-25.5$ & .0021 \\
MCA lesions total & 16 & 0 & 62 & $2.5-44.5$ & .0009 \\
MCA volume left & 889.5 & 0 & 8760 & $0-3678$ & $<.0001$ \\
MCA volume right & 580.5 & 0 & 7516 & $113-7701.5$ & $<.0001$ \\
MCA volume total & 1982.5 & 0 & 16,276 & $0-7.5$ & $<.0001$ \\
PCA lesions left & 2 & 0 & 45 & $0-8.5$ & $<.0001$ \\
PCA lesions right & 3 & 0 & 23 & $0-14$ & $<.0001$ \\
PCA lesions total & 5 & 0 & 51 & $0-1403.5$ & $<.0001$ \\
PCA volume left & 132.5 & 0 & 2334 & $0-1112.5$ & $<.0001$ \\
PCA volume right & 171 & 0 & 2492 & $0-2308.5$ & $<.0001$ \\
PCA volume total & 418.5 & 0 & 3625 & & $<.0001$ \\
\hline
\end{tabular}

Abbreviations: ACA, anterior cerebral artery; MCA, middle cerebral artery; PCA, posterior cerebral artery.

*Normal distribution assessed with the Kogomorov-Smirnov Z test.

\section{WMH Volume and Lesion of ACA-MCA-PCA}

The $\mathrm{WMH}$ volume and the number of lesions were quantified for each vascular territory (ACA-MCAPCA) in the left and right hemispheres of the patients, and the results are given in Table 3 . We compared the $\mathrm{WMH}$ volume and number of lesions of the right and left sides, and the Wilcoxon test showed a statistically significant difference in the MCA territory for the volume of the lesions (median volume of WMH on the left side $=889.5 \mathrm{~mm}^{3}$; median volume of WMH on the right side $\left.=580.5 \mathrm{~mm}^{3} ; P=.0416\right) ;$ no statistically significant difference was found with the other territories and by considering the number of lesions (Table 4). Box plots are given in Figure 1.
WMH Volume and Lesion of Deep White Matter Hyperintensity (DWMH)-Periventricular White Matter Hyperintensity (PVWMH)

We also explored the WMH volume of DWMH and PVWHM in the left and right hemispheres of the patients, and the results are given in Table 5. The Wilcoxon analysis found a statistically significant difference between the volume of the DWMH and PVWMH $(P=.001)$, whereas no statistically significant difference was found in the DWMH volume between the left and right sides $(P=.9206)$. On the contrary, a statistically significant difference was found for the PVWMH volume $(P=.0088)$ between the left and right sides. Box plots are given in Figure 2.

Table 4. Wilcoxon analysis of WMH volume and number of lesions in ACA-MCA-PCA

\begin{tabular}{lcccccc}
\hline & \multicolumn{2}{c}{ Right side } & & \multicolumn{2}{c}{ Left side } \\
\cline { 2 - 3 } & Median & Interquartile range & & Median & Interquartile range & Wilcoxon test \\
\hline ACA volume $\left(\mathrm{mm}^{3}\right)$ & 242.5 & $82.5-511.5$ & & 282.5 & $72.5-658.5$ & .3877 \\
MCA volume $\left(\mathrm{mm}^{3}\right)$ & 580.5 & $267.5-1791.5$ & & 889.5 & $317-2377$ & $.0416 *$ \\
PCA volume $\left(\mathrm{mm}^{3}\right)$ & 171 & $0-433$ & & 132.5 & $0-437$ & .8294 \\
ACA number of lesions & 4 & $2-7$ & & 4 & $1.5-7$ & .7321 \\
MCA number of lesions & 7 & $4-15$ & & 16 & $4-12.5$ & .1809 \\
PCA number of lesions & 3 & $0-4$ & & $0-4$ & .1607 \\
\hline
\end{tabular}

Abbreviations: ACA, anterior cerebral artery; MCA, middle cerebral artery; PCA, posterior cerebral artery.

*Statistically significant $P$ value. 

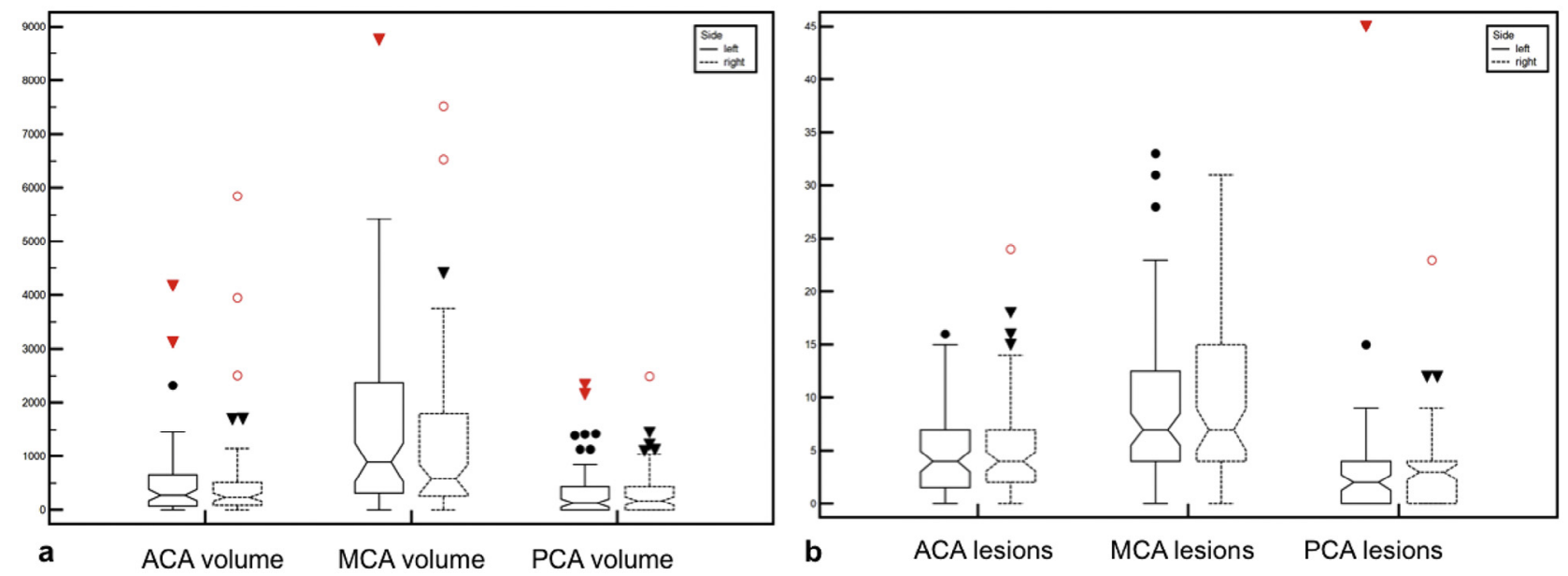

Figure 1. Box plot that shows the volume (A) and number of lesions (B) of WMH for the ACA-MCA-PCA territories on the right and left sides. Abbreviations: ACA, anterior cerebral artery; MCA, middle cerebral artery; PCA, posterior cerebral artery; WMH, white matter hyperintensity.

\section{Discussion}

In this study our purpose was to assess the volumetric distribution of the WMHs in the left and right hemispheres according to the different arterial territories and to the involvement of the periventricular or deep white matter.

In pathological studies WMHs are often seen together with vessels affected by small vessel disease. ${ }^{22}$ The mechanism of damage is still debated, and it is hypothesized that the chronic hypoperfusion of the white matter and the concomitant disruption of the blood-brain barrier could lead to chronic leakage of plasma into the white matter with a consequent damage. ${ }^{23,24}$

We found that the median WMH volume was $3505.5 \mathrm{~mm}^{3}$ with values ranging from 0 to 28,324 ; these $\mathrm{WMH}$ values are lower compared with those found by De Carli et $\mathrm{al}^{1}$ in 2005, where WMH volumes ranged from 1100 to $63,000 \mathrm{~mm}^{3}$. However, this difference could be explained with the fact that the authors assessed patients in their 70s who presented to a specialty clinic (Alzheimer), suggesting that white matter lesions in their sample were at an advanced stage, whereas we enrolled a younger population (subjects aged more than 40 years) who underwent brain MRI because of the pres- ence of atherosclerotic disease in the carotid arteries detected by ultrasonography. In a study performed by Wen et $\mathrm{al}^{24}$ to assess the relationship between brain atrophy and WMHs in 394 patients, the WMH volumes ranged from 352 to $50,408 \mathrm{~mm}^{3}$; also in this case the difference in WMHs could be explained with the patients' age, which ranged from 60 to 64 years. It is interesting to observe that also in this paper the WMH volume was not distributed according to a Gaussian distribution and that it was strongly skewed toward the lower end.

In our population, the median $\mathrm{WMH}$ volume was $1289 \mathrm{~mm}^{3}$ in the right hemisphere and $1760 \mathrm{~mm}^{3}$ in the left hemisphere $(P=.0899)$. These values did not show a statistically significant association, but the $P$ value $<.1$ could be a statistical trend. The fact that the left hemisphere suffers a bigger burden of WMHs is concordant with 2 previous observations: (1) the chronic tissue damage may occur in a subset of individuals with $\geq 70 \%$ ICA stenosis, globally exhibiting more extensive WMHs ipsilateral to the ICA stenosis ${ }^{25}$ and (2) the carotid atherosclerotic plaque size and composition are not symmetrically distributed and the features related to the plaque's vulnerability (e.g., intraplaque hemorrhage) are more likely found in the left-sided plaques. ${ }^{17}$ These observations strengthen the model where the WMHs could be related/

Table 5. Summary of WMH volume for DWMH and PVWMH

\begin{tabular}{lccrr}
\hline & Median & Minimum & Maximum & 5-95 percentiles \\
\hline PVWMH left & 1319 & 0 & 11,353 & $31.500-5510.500$ \\
PVWMH right & 740.5 & 0 & 8000 & $.000-3959.000$ \\
PVWMH total & 2078 & 0 & 19,353 & $36.500-9149.500$ \\
DWMH left & 448.5 & 0 & 2016 & $13.500-1754.500$ \\
DWMH right & 389 & 0 & 6400 & $10.000-1648.500$ \\
DWMH total & 987 & 0 & 8319 & $32.000-2978.500$ \\
\hline
\end{tabular}

Abbreviations: DWMH, deep white matter hyperintensity; PVWMH, periventricular white matter hyperintensity. 


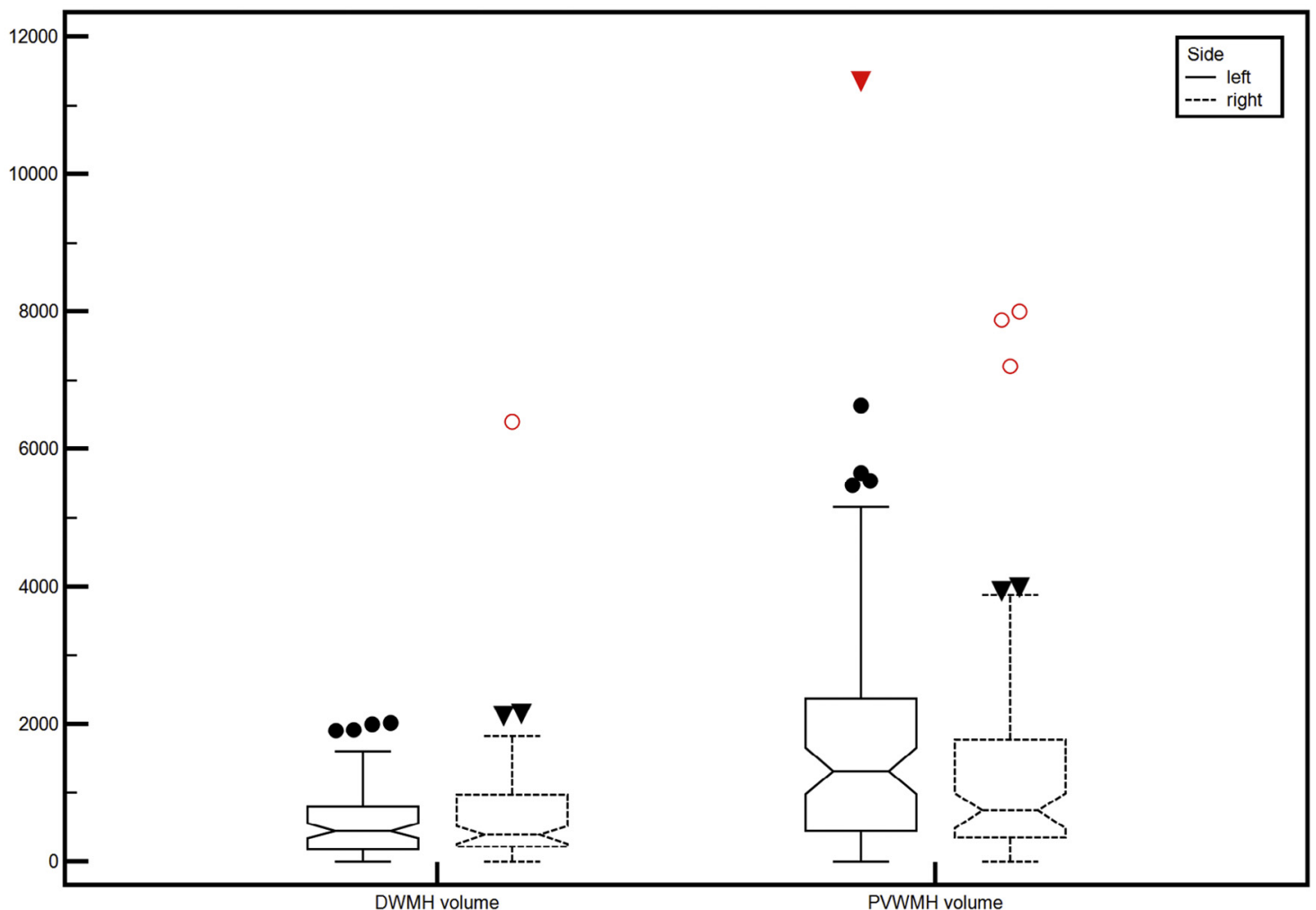

Figure 2. Box plot that shows the DWMH and PVWMH on the right and left sides. Abbreviations: DWMH, deep white matter hyperintensity; PVWMH, periventricular white matter hyperintensity.

associated to the pathology of the carotid arteries. These results are also concordant with previously published studies by Saba et al. ${ }^{26-28}$

For each vascular territory we assessed the WMH volume and the number of lesions in the left and right hemispheres, and we found a statistically significant difference in the MCA territory for the volume of WMHs $(P=.0416)$, whereas no statistically significant difference was found with the other territories for the WMHs and by considering the number of lesions. This difference in the WMH volume of the MCA between the 2 sides, not significant for the ACA and PCA territories, is interesting, suggesting that in the MCA territories the effect of the carotid artery pathologies could be emphasized. Another option could be that the MCA territory is the biggest vascular territory and therefore a statistical effect that maximizes the difference compared with the ACA and PCA territories could be present. WMHs are frequently found together with lacunar infarcts, and these conditions are usually thought to be concomitant expressions of arteriolosclerotic disease ${ }^{29}$; but our results suggest that WMHs may also represent something else besides the generally accepted concept of small vessel disease, as indicated by other studies. ${ }^{30}$ From Table 3 we can see that the median value of the WMHs in the ACA, MCA, and PCA were 538, 1982, $418 \mathrm{~mm}^{3}$. The propor- tion of these values is quite similar to those found in other studies with a slightly lower WMH volume. ${ }^{24}$

We also explored the WMH volume of DWMH and PVWHM in the left and right hemispheres, and we found a statistically significant difference between the volume of the DWMH and PVWMH $(P=.001)$, whereas no statistically significant difference was found in the DWMH volume between the left and right sides $(P=.9206)$. On the contrary, a statistically significant difference was found for the PVWMH volume $(P=.0088)$ between the left and right sides. The difference in the PVWMH volume between the left and right sides and the absence of difference of the DWMH seems to strengthen the hypothesis of a different etiopathogenesis for these 2 conditions. We found that DWMH accounted for about one third of the total WMH volume, with the other two thirds being PVWMH, and these results are similar to those observed in another study $^{31}$ that showed that DWMH had a stronger relationship with cortical perfusion. It is important to underline that we tested the effect of the side (right/left) of the WMH lesion volume in a population with a carotid artery stenosis degree $>30 \%$. The presence of stenosis degree was considered a parameter related to the presence of WMHs, ${ }^{32}$ but further studies are needed to test the effect of the relationship between $\mathrm{WMH}$ volume, size, and carotid artery plaque composition. . $^{33,34}$ 
In this study the potential chronic silent brain infarcts, which are a common source of confounding in WMH studies, were considered, and an effort to differentiate WMHs from possible chronic silent brain infarcts was performed.

In this study there are some limitations. First, it's retrospective in nature. A prospective longitudinal study is needed to validate our observations. However, we think that our data were not biased because the methodology of patient selection was homogeneous. A second limitation could be considered the brain vascular anatomy due to its frequent anatomical variations that could introduce a bias in the study; we think that this could be considered a minor limitation. Third, a homogenous patient selection does not guarantee the absence of bias; although selection bias may be limited, information bias or confounding may still significantly be present.

\section{Conclusion}

In conclusion, results of our study suggest that there is a difference in the distribution of WMHs in the brain hemispheres according to the left/right side in the MCA territories and for the PVWM.

\section{References}

1. DeCarli C, Fletcher E, Ramey V, et al. Anatomical mapping of white matter hyperintensities (WMH): exploring the relationships between periventricular $\mathrm{WMH}$, deep WMH, and total WMH burden. Stroke 2005;36:50-55.

2. Ylikoski A, Erkinjuntti T, Raininko R, et al. White matter hyperintensities on MRI in the neurologically nondiseased elderly. Analysis of cohorts of consecutive subjects aged 55 to 85 years living at home. Stroke 1995;26:1171-1177.

3. Awad IA, Spetzler RF, Hodak JA, et al. Incidental subcortical lesions identified on imaging in the elderly. I. Correlation with age and cerebrovascular risk factors. Stroke 1986;17:1084-1089.

4. Pantoni L, Garcia JH. Pathogenesis of leukoaraiosis: a review. Stroke 1997;28:652-659.

5. Fazekas F, Kleinert R, Offenbacher H, et al. Pathologic correlates of incidental MRI white matter signal hyperintensities. Neurology 1993;43:1683-1689.

6. Aribisala BS, Morris Z, Eadie E, et al. Blood pressure, internal carotid artery flow parameters, and age-related white matter hyperintensities. Hypertension 2014;63:10111018.

7. Gąsecki D, Kwarciany M, Nyka W, et al. Hypertension, brain damage and cognitive decline. Curr Hypertens Rep 2013;15:547-558. doi:10.1007/s11906-013-0398-4.

8. Purkayastha S, Fadar O, Mehregan A, et al. Impaired cerebrovascular hemodynamics are associated with cerebral white matter damage. J Cereb Blood Flow Metab 2014;34:228-234.

9. Mortamais M, Artero S, Ritchie K. Cerebral white matter hyperintensities in the prediction of cognitive decline and incident dementia. Int Rev Psychiatry 2013;25:686-698.

10. Park JH, Seo SW, Kim C, et al. Effects of cerebrovascular disease and amyloid beta burden on cognition in subjects with subcortical vascular cognitive impairment. Neurobiol Aging 2014;35:254-260.
11. Scheltens P, Barkhof F, Leys D, et al. A semiquantative rating scale for the assessment of signal hyperintensities on magnetic resonance imaging. J Neurol Sci 1993;114:7-12.

12. Wang R, Li C, Wang J, et al. Automatic segmentation and quantitative analysis of white matter hyperintensities on FLAIR images using trimmed-likelihood estimator. Acad Radiol 2014;doi:10.1016/j.acra.2014.07.001. PMID: 25176451. pii: S1076-6332(14)00245-1, Epub ahead of print.

13. Gibson E, Gao F, Black SE, et al. Automatic segmentation of white matter hyperintensities in the elderly using FLAIR images at 3T. J Magn Reson Imaging 2010;31:13111322.

14. Naess H, Waje-Andreassen U, Thomassen L, et al. High incidence of infarction in the left cerebral hemisphere among young adults. J Stroke Cerebrovasc Dis 2006;15:241-244.

15. Foerch C, Misselwitz B, Sitzer M, et al. Difference in recognition of right and left hemispheric stroke. Lancet 2005;366:392-393.

16. Hedna VS, Bodhit AN, Ansari S, et al. Hemispheric differences in ischemic stroke: is left-hemisphere stroke more common? J Clin Neurol 2013;9:97-102.

17. Selwaness $M$, van den Bouwhuijsen $Q$, van Onkelen RS, et al. Atherosclerotic plaque in the left carotid artery is more vulnerable than in the right. Stroke 2014;45:32263230. PMID: 25228259. pii: STROKEAHA.114.005202, Epub ahead of print.

18. Barnett HJ, Taylor DW, Eliasziw M, et al. Benefit of carotid endarterectomy in patients with symptomatic moderate or severe stenosis. N Engl J Med 1998;339:1415-1425.

19. Saba L, Sanfilippo R, Porcu M, et al. Relationship between white matter hyperintensities volume and the circle of Willis configurations in patients with carotid artery pathology. Eur J Radiol 2017 Apr;89:111-116. doi:10.1016/ j.ejrad.2017.01.031. [Epub 2017 Feb 1]; PubMed PMID: 28267525.

20. Vermeer SE, Longstreth WT Jr, Koudstaal PJ. Silent brain infarcts: a systematic review. Lancet Neurol 2007;6:611-619.

21. Jeerakathil T, Wolf PA, Beiser A, et al. Cerebral microbleeds: prevalence and associations with cardiovascular risk factors in the Framingham Study. Stroke 2004;35:1831-1835.

22. O'Sullivan M, Lythgoe DJ, Pereira AC, et al. Patterns of cerebral blood flow reduction in patients with ischemic leukoaraiosis. Neurology 2002;59:321-326.

23. Topakian R, Barrick TR, Howe FA, et al. Blood-brain barrier permeability is increased in normal-appearing white matter in patients with lacunar stroke and leucoaraiosis. J Neurol Neurosurg Psychiatry 2010;81:192197.

24. Wen W, Sachdev PS, Chen X, et al. Gray matter reduction is correlated with white matter hyperintensity volume: a voxel-based morphometric study in a large epidemiological sample. Neuroimage 2006;29:1031-1039. [Epub 2005 Oct 25]; PMID: 16253521.

25. Enzinger C, Ropele S, Gattringer T, et al. High-grade internal carotid artery stenosis and chronic brain damage: a volumetric magnetic resonance imaging study. Cerebrovasc Dis 2010;30:540-546.

26. Saba L, Raz E, Grassi R, et al. Association between the volume of carotid artery plaque and its subcomponents and the volume of white matter lesions in patients selected for endarterectomy. AJR Am J Roentgenol 2013;201:W747-W752. doi:10.2214/AJR.12.10217. PMID: 24147504.

27. Saba L, Pascalis L, Sanfilippo R, et al. Carotid artery wall thickness and leukoaraiosis: preliminary results using 
multidetector row CT angiography. AJNR Am J Neuroradiol 2011;32:955-961. doi:10.3174/ajnr.A2396. [Epub 2011 Feb 24]; PMID: 21349963.

28. Saba L, Sanfilippo R, Pascalis L, et al. Carotid artery abnormalities and leukoaraiosis in elderly patients: evaluation with MDCT. AJR Am J Roentgenol 2009;192:W63-W70. doi:10.2214/AJR.07.3566. PMID: 19155382.

29. Chen X, Wen W, Anstey KJ, et al. Prevalence, incidence, and risk factors of lacunar infarcts in a community sample. Neurology 2009;73:266-272.

30. Debette S, Beiser A, DeCarli C, et al. Association of MRI markers of vascular brain injury with incident stroke, mild cognitive impairment, dementia, and mortality: the Framingham Offspring Study. Stroke 2010;41:600-606.
31. Wen W, Sachdev P, Shnier R, et al. Effect of white matter hyper-intensities on cortical cerebral blood volume using perfusion MRI. Neuroimage 2004;21:1350-1356.

32. Demirtaş H, Özkaynak C, Durmaz MS. Leukoaraiosis and carotid artery stenosis: evaluation with CT angiography. J Comput Assist Tomogr 2013;37:327-332.

33. Altaf N, Morgan PS, Moody A, et al. Brain white matter hyperintensities are associated with carotid intraplaque hemorrhage. Radiology 2008;248:202-209. doi:10.1148/ radiol.2481070300. PMID: 18566173.

34. Saba L, Yuan C, Hatsukami TS, et al. Carotid artery wall imaging: perspective and guidelines from the ASNR vessel wall imaging study group and expert consensus recommendations of the American Society of Neuroradiology. AJNR Am J Neuroradiol 2018;39:E9-E31. 